\title{
A capillary device for growing large protein crystals
}

Koji Inaka ${ }^{a}$, Sachiko Takahashi ${ }^{\mathrm{b}}$, Bin Yan $^{\mathrm{b}}$, Misako Koga ${ }^{\mathrm{b}}$, Yoshinobu Hashizume ${ }^{\mathrm{b}}$, Masayuki Kamo $^{a}$, Naoki Furubayashi ${ }^{a}$ and Hiroaki Tanaka ${ }^{b}$

aMaruwa Foods and Biosciences Inc., 170-1 Tsutsui-cho, Yamatokoriyama, Nara 639-1123 JAPAN, crystal@maruwafoods.jp

${ }^{\mathrm{b} C o n f o c a l}$ Science Inc., 2-12-2 Iwamoto-cho, Chiytoda-ku, Tokyo 101-0023 JAPAN, info@confsci.co.jp

Neutron diffraction experiment is very useful ${ }^{1)}$, but it is difficult to grow suitable crystals for neutron diffraction experiment because it requires much larger crystals if compared to the crystal size for $\mathrm{X}$-ray crystallography (more than 1000 times in volume).

Here we introduce a capillary device especially for growing large protein crystals ${ }^{2)}$. The main feature is that it includes self-contained dialysis membrane which provides dialysis method in the capillary. In the poster, we will introduce the preparation methods and large crystals which grew in this device.

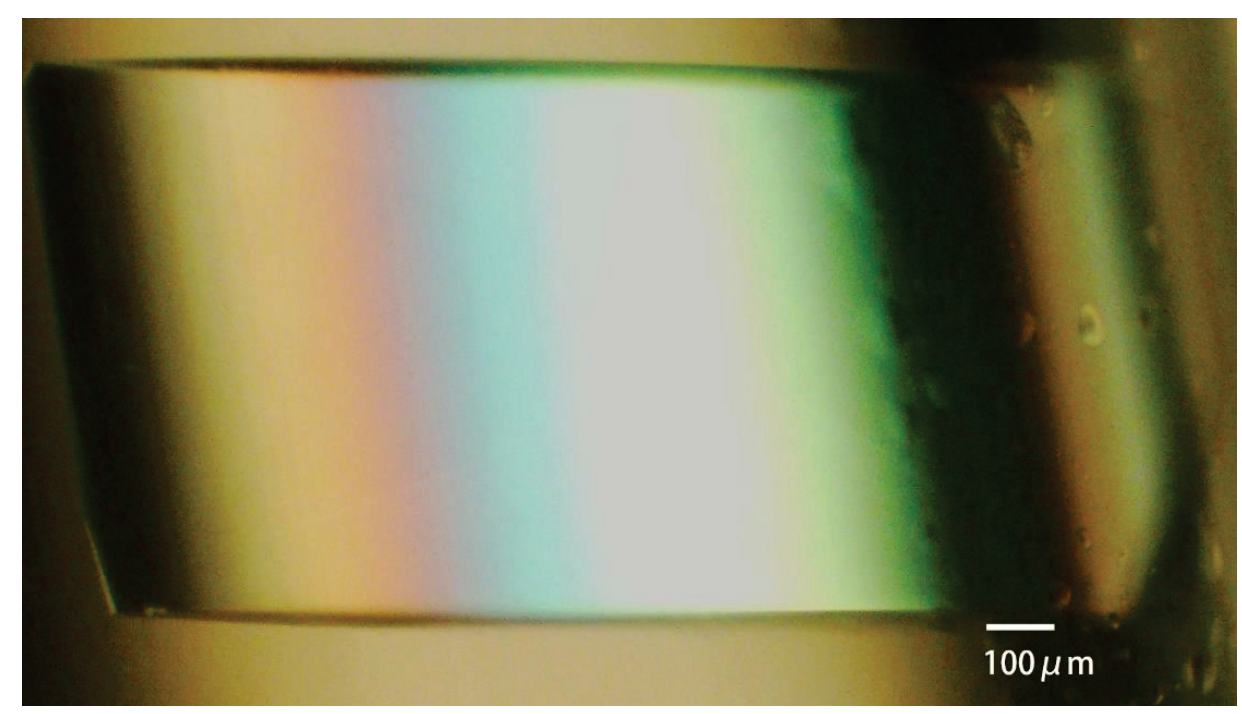

Figure 1. A large lysozyme crystal grown in the capillary device

\section{References}

1) J. D. Ng et al. Acta Cryst. (2015). F71(4), 358-370.

2) Patent number: P6473788 (2019/02/01) (Japan) 